\title{
Investigation of 3D Printed Electrically Small Folded Spherical Meander Wire Antenna
}

\author{
Myeongjun Kong • Geonyeong Shin · Su-Hyeon Lee · Ick-Jae Yoon*
}

\begin{abstract}
The radiation properties and fabrication precautions of a 3D printed, electrically small folded spherical meander wire monopole antenna are investigated. The antenna is self-resonant and shows sufficiently high radiation efficiency at an electrical size $k a$ of 0.4 , with the radiation quality factor $Q$ approaching the lower physical bound. In antenna fabrication, the possible structural deformation due to gravity is examined before the antenna frame is $3 \mathrm{D}$-printed. The required conductivity is achieved by multiple manual paintings of a silver paste. The radiation efficiency and pattern show very good agreement with the computed expectations, whereas the resonant frequency deviates by $11.8 \%$. The method to minimize such a fabrication error when using $3 \mathrm{D}$ printing technology for wire antennas is discussed.
\end{abstract}

Key Words: 3D Printing Technology, Electrically Small Antennas, Spherical Meander Antennas, Structural Deformation Analysis.

\section{INTRODUCTION}

Rapidly developing 3D printing (3DP) technology is being widely used in scientific and engineering areas [1-3]. Such progress has attracted the interest of the antenna community, and several existent antenna designs created with the aid of the technology have been verified, including horn, waveguide, lens, reflectarray, patch, wire antennas, only to mention a few [4-9]. In addition, novel electrically small antenna (ESA) designs have been proposed that might be difficult to verify experimentally without 3DP technology $[10,11]$. In extending this line of research, it is worth further exploring 3DP technology to examine its potential to improve radiation properties and to review its instructions for antenna fabrication.

In this paper, we demonstrate the impedance and radiation properties of an electrically small folded spherical meander (FSM) wire monopole antenna built using a selective laser sintering (SLS) 3DP technology and manual painting of a silver paste. The FSM design itself is not new but has been reported in [12] as a printed form on a hemispherical dielectric surface. The meander design is prevalent due to its intuitiveness in achieving a small form factor. It has been found that the $3 \mathrm{D}$ printed FSM antenna shows superior radiation efficiency when compared with the antenna in [12] due to the advantageous 3DP manufacturing technology. Some preliminary simulation results are shown in [13]. In this paper, further description of the antenna design, structural stability analysis due to the gravity, fabrication process, and the measured results are presented. In addition, a more comprehensive study of the use of 3DP technology and manual metallic painting for wire antenna fabrication is provided. The method to reduce fabrication error when using the proposed technology is also discussed.

\section{ANTENNA DESIGN}

The FSM wire monopole antenna on an infinite ground pla-

Manuscript received August 7, 2017 ; Revised September 20, 2017 ; Accepted September 24, 2017. (ID No. 20170807-036J)

Department of Electrical Engineering, Chungnam National University, Daejeon, Korea.

"Corresponding Author: Ick-Jae Yoon (e-mail: ijyoon@cnu.ac.kr)

This is an Open-Access article distributed under the terms of the Creative Commons Attribution Non-Commercial License (http://creativecommons.org/licenses/by-nc/4.0) which permits unrestricted non-commercial use, distribution, and reproduction in any medium, provided the original work is properly cited.

(c) Copyright The Korean Institute of Electromagnetic Engineering and Science. All Rights Reserved. 
ne is shown in Fig. 1. It is composed of multiple hemispherical meander arms connected at the top. The single arm portion is marked by dashed lines in Fig. 1(a). We use Numerical Electromagnetics Code (NEC) for the antenna design. The design values are $\alpha=77.8^{\circ}$ (the angle representing the arc length in a single meander arm; see Fig. 1(a)), $\beta=6^{\circ}$ (the angle between the parallel sections in a single meander arm; see Fig. $1(\mathrm{~b})), D=1$ $\mathrm{mm}$ (the wire diameter), $a=25.46 \mathrm{~mm}$ (the radius of the imaginary sphere enclosing the antenna, including $D$ ), and $n=4$ (the number of meander arms) for impedance matching and high radiation efficiency. The available values of the design parameters are limited by the structural aspects and the resolution of the existent 3DP technology. For example, if the desired $n$ is four for stepping up the small radiation resistance to a proper value for high radiation efficiency, the maximum value of $\alpha$ should be less than $90^{\circ}$ to avoid any physical interference between the wires. Likewise, the wire diameter $D$ thinner than 0.8 $\mathrm{mm}$ is hardly utilized because the resolution of the SLS method of 3DP using a polyamide material is usually $0.8 \mathrm{~mm}$ (or even thicker).

The antenna is made of copper, and the simulation results in Fig. 2 show that the antenna resonates at $750 \mathrm{MHz}$ with radiation efficiency of $96 \%$. The electrical size $k a$, where $k$ is a wave propagation constant in free space, is 0.4 . The quality factor $Q$ of the designed antenna is 26.03 , approaching the lower physical bound of electrically small electric antennas at a given electrical size [14]. It is found that the radiation efficiency is improved when compared with the antenna in [12], which is built by printing silver nanoparticle ink on a hemispherical dielectric surface. It shows $71 \%$ radiation efficiency at $k a$ of 0.46 . Such improvement is because thick wires can be utilized in the air, and no lossy dielectric is present in the FSM wire antenna. It is found that the high radiation efficiency characteristic of the FSM wire antenna is maintained, even when the copper is replaced by a less conductive material in NEC simulation, as shown by the dashed line in Fig. 2. It is checked because commercially available conductive paint and spray would show lower

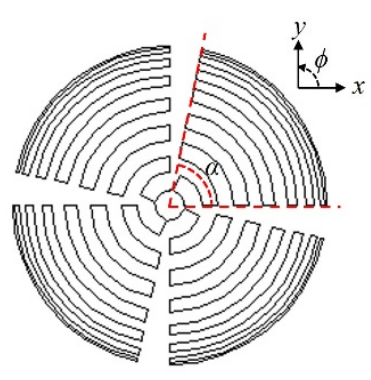

(a)

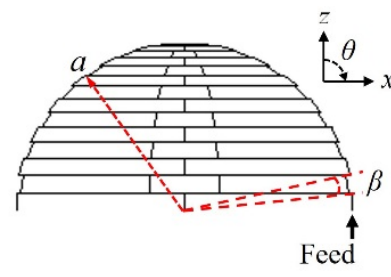

(b)
Fig. 1. Folded spherical meander (FSM) wire monopole antenna: (a) top view and (b) side view. The infinite ground plane is not described.

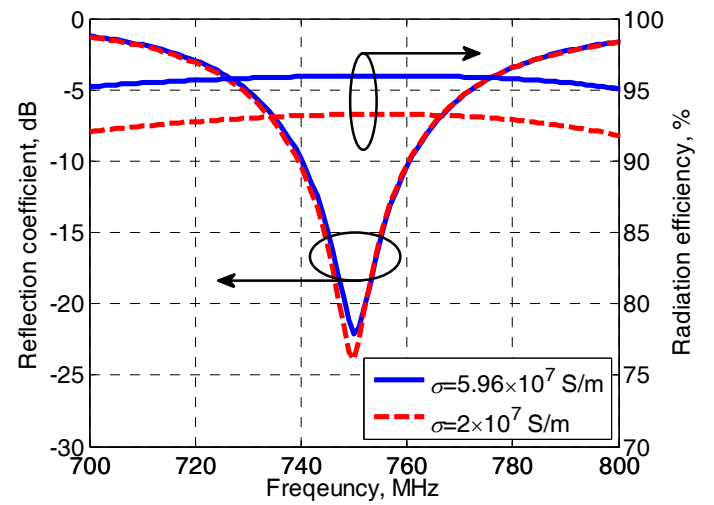

Fig. 2. Simulated reflection coefficient and radiation efficiency of the FSM monopole antenna ( $\sigma$ : conductivity).

conductivity than bulk copper.

\section{ANTENNA FABRICATION}

For the fabrication, we first carry out a structural deformation analysis due to the gravity using OptiStruct (Altair Engineering Inc., Troy, MI, USA) as shown in Fig. 3(a). The original antenna structure and the deformed one are described by a solid black

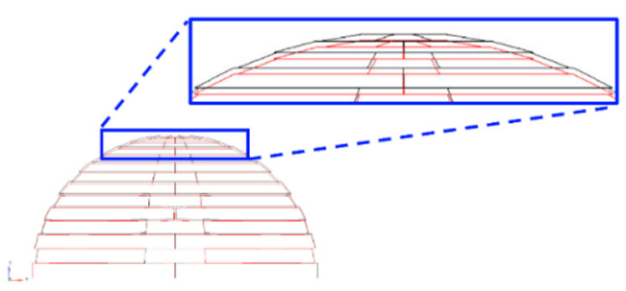

(a)

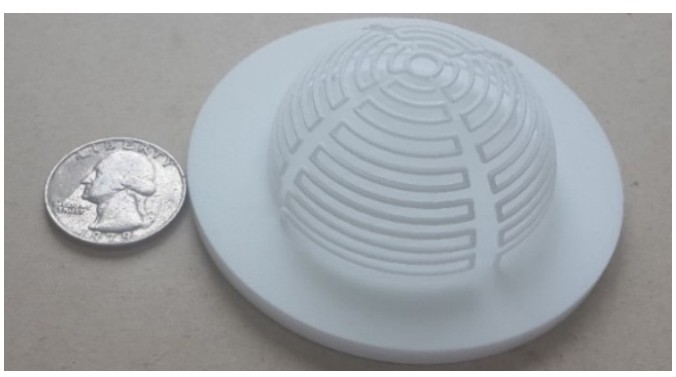

(b)

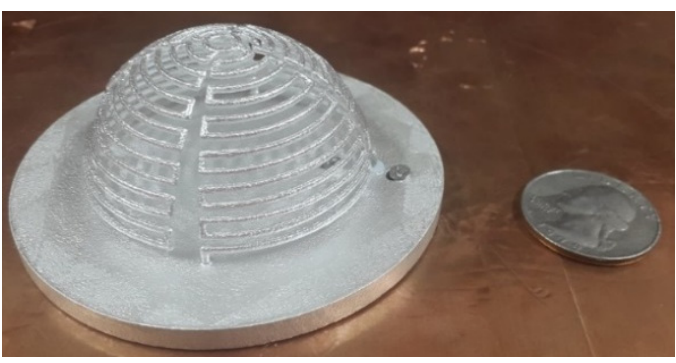

(c)

Fig. 3. Antenna fabrication. (a) Structural deformation analysis, (b) $3 \mathrm{D}$ printed frame of the antenna, and (c) fabricated antenna after silver painting (final). 
line and a solid red line, respectively. The frame is made of a polyamide material of PA2200. The maximum deformation occurs at the top and is $0.377 \mathrm{~mm}$. It is about a $1.5 \%$ deviation from the antenna height and would have a negligible effect on the antenna performance. It is worth noting that the antenna does not contain any additional supporters to hold it, being different from other wire antennas built using 3DP technology in $[10,11]$. Such a supporter-less frame could make plating or the metal painting process easier and faster. The antenna frame and a partial circular ground plane are printed together using an SLS type EOS P395 3D printer with the same material. The height and radius of the partial ground are $4.5 \mathrm{~mm}$ and $38.19 \mathrm{~mm}$, respectively, which has a negligible effect on the antenna performance because its electrical dimension is much smaller than the wavelength. The printed object is shown in Fig. 3(b) [13]. It is then brushed with a silver conductive paint from Electrolube that has a surface resistance of $0.01-0.03 \Omega /$ sq with a drying time of 10 minutes at $20^{\circ} \mathrm{C}$ when deposited over a thickness of $0.6-2 \mathrm{~g} / 100 \mathrm{~cm}^{2}$ [15]. The painting is repeated five times until achieving sufficiently high conductivity. Afterwards, it is installed on a large ground plane for measurement, as shown in Fig. 3(c). A US quarter is placed in the photos as a size reference.

\section{ANTENNA MEASUREMENT}

We measure the resonant frequency and the radiation efficiency of the fabricated antenna upon each painting of the silver paste, as shown in Fig. 4(a). The radiation efficiency is measured using the Wheeler cap method. It is found that the radiation efficiency increases with the number of paintings because electrical conductivity increases with thicker and drier silver paste distribution. We also observed that the measured resonant frequency shows a gradual increase per painting. This is strongly related to the impedance characteristic of the FSM wire antenna, which is very sensitive to the wire thickness $D$. As shown in Fig. 4(b), the resonant frequency shifts sensitively with even small variations in $D$. The resonant frequency is finally measured as $838.9 \mathrm{MHz}$, which is shifted upward by $88.9 \mathrm{MHz}$ or $11.8 \%$ from the simulation $(D=1 \mathrm{~mm})$. We find that the wire diameter of the built antenna in Fig. 3(c) is about $1.4 \mathrm{~mm}$. The simulated resonant frequency is $844 \mathrm{MHz}$ when $D$ is $1.4 \mathrm{~mm}$, which is very close to the measurement. The diameter of the printed wire before metallization is approximately $1 \mathrm{~mm}$ all around the structure, and it can be inferred that the painting process adds a thickness of $0.4 \mathrm{~mm}$. This experiment highlights the fact that the thickness of the painted metal layer must be considered and the plastic frame of the antenna should intentionally be printed thinner. This is because the conductivity of most commercial metallic paint or spray is low when distributed in a thin single layer but needs sufficient thickness to achieve high conductivity.
The final measured radiation efficiency is $93.8 \%$, verifying the simulation.

The normalized $\theta$-sweep radiation patterns when $\phi=0^{\circ}$ are shown in Fig. 4(c). The simulation assumes an infinitely large ground plane, whereas an $88 \mathrm{~cm} \times 88 \mathrm{~cm}$ one is utilized in the measurement. It is found from the simulated result that the null point of the omni-directional pattern occurs at $-2^{\circ}$. This is caused by the slightly non-uniform current distribution of the FSM wire antenna. Such a phenomenon can occur in folded monopole antennas when the length of each arm is away from

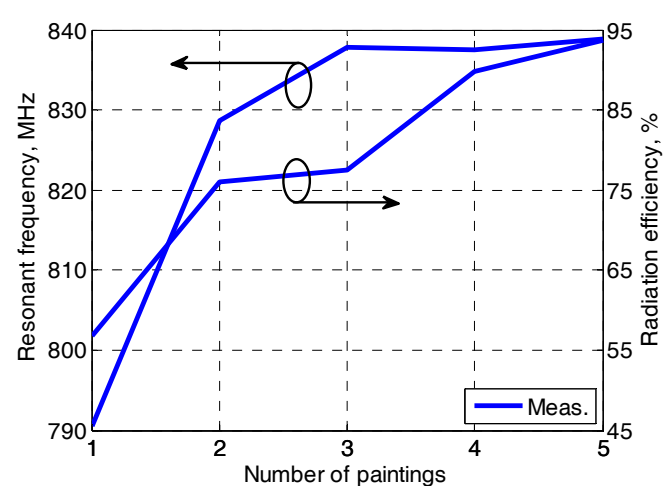

(a)

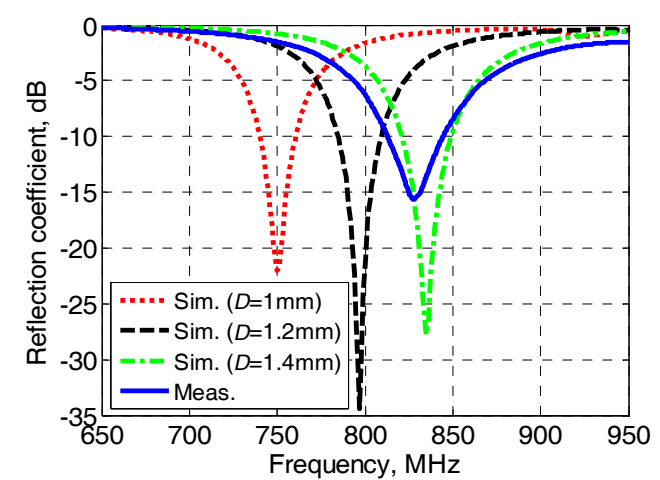

(b)

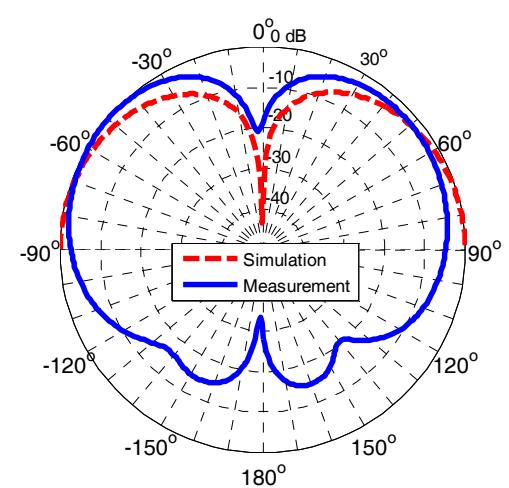

(c)

Fig. 4. Simulated and measured results. (a) Measured resonant frequency and radiation efficiency per the number of paintings, (b) simulated and measured reflection coefficients, and (c) simulated and measured normalized $\theta$-sweep radiation patterns $\left(\phi=0^{\circ}\right)$. 
$0.25 \lambda[16]$. The total length of the single meander arm in the designed FSM wire monopole antenna is $0.86 \lambda$. The tilted pattern is also clearly observed in the measurement, corroborating the usefulness of the 3DP technology with manual metallic painting for rapid and easy experimental verification.

\section{CONCLUSION}

In this paper, we designed an electrically small FSM wire monopole antenna and demonstrated the radiation properties after building it using SLS 3DP technology and silver paste painting. The built FSM wire antenna does not contain any additional supporters to hold the 3D printed plastic frame. This kind of fabrication was determined by a structural deformation analysis before the 3D printing and accelerated the whole fabrication process, including the silver painting. In terms of measurement, it was found that the resonant frequency deviates from the simulation by $11.8 \%$ due to the difference in wire thickness between the simulation and fabrication models, which occurred during the multiple metal paintings for high conductivity. Therefore, it is necessary to consider the space for the metal to be deposited when printing the frame. Nevertheless, based on the measurement it was observed that the radiation efficiency and pattern show good agreement with the simulation. Furthermore, when compared with the similar volumetric meander antenna design in [12], which was made using conformal printing technology, the proposed 3D printed FSM wire antenna shows superior radiation efficiency. Currently, 3DP technology has limitations in terms of its resolution and build quality, especially when metallization is considered. However, it could be effectively used as a means of easy verification of antenna performance to a certain degree.

This research was supported by Chungnam National University, Daejeon, Republic of Korea (No. 2016-1780-01).

\section{REFERENCES}

[1] I. Gibson, D. Rosen, and B. Stucker, Additive Manufacturing Technologies: 3D Printing, Rapid Prototyping and Direct Digital Manufacturing. New York, NY: Springer, 2015.

[2] B. C. Gross, J. L. Erkal, S. Y. Lockwood, C. Chen, and D. M. Spence, "Evaluation of 3D printing and its potential impact on biotechnology and the chemical sciences," Analytical Chemistry, vol. 86, no. 7, pp. 3240-3253, 2014.

[3] J. Lee, J. Bang, and J. Choi, "Realistic head phantom for evaluation of brain stroke localization methods using 3D printer," Journal of Electromagnetic Engineering and Science, vol. 16, no. 4, pp. 254-258, 2016.
[4] J. C. S. Chieh, B. Dick, S. Loui, and J. D. Rockway, "Development of a $\mathrm{Ku}$-band corrugated conical horn using 3-D print technology," IEEE Antennas and Wireless Propagation Letters, vol. 13, pp. 201-204, 2014.

[5] G. L. Huang, T. H. Chio, T. S. Yeo, and S. G. Zhou, "Application of 3-D metal-direct-printing technique for waveguide antenna fabrication," in Proceedings of 2015 IEEE International Symposium on Antenna and Propagation (APSURSI), Vancouver, Canada, 2015, pp. 316-317.

[6] M. Liang, W. R. Ng, K. Chang, K. Gbele, M. E. Gehm, and H. Xin, "A 3-D Luneburg lens antenna fabricated by polymer jetting rapid prototyping," IEEE Transaction on Antennas and Propagation, vol. 62, no. 4, pp. 1799-1807, 2014.

[7] P. Nayeri, M. Liang, R. A. Sabory-Garcia, M. Tuo, F. Yang, M. Gehm, H. Xin, and A. Z. Elsherbeni, "3D printed dielectric reflectarrays: low-cost high-gain antennas at sub-millimeter waves," IEEE Transaction on Antennas and Propagation, vol. 62, no. 4, pp. 2000-2008, 2014.

[8] S. Moscato, R. Bahr, T. Le, M. Pasian, M. Bozzi, L. Perregrini, and M. M. Tentzeris, "Infill-dependent 3-Dprinted material based on NinjaFlex filament for antenna applications," IEEE Antennas and Wireless Propagation Letters, vol. 15, pp. 1506-1509, 2016.

[9] H. Huang, K. Nieman, P. Y. Chen, M. Ferrari, Y. Hu, and D. Akinwande "Properties and applications of electrically small folded ellipsoidal helix antenna," IEEE Antennas and Wireless Propagation Letters, vol. 11, pp. 678-681, 2012.

[10] O. S. Kim, "Rapid prototyping of electrically small spherical wire antennas," IEEE Transaction on Antennas and Propagation, vol. 62, no. 7, pp. 3839-3842, 2014.

[11] M. Kong, G. Shin, S. H. Lee, and I. J. Yoon, "Electrically small folded spherical helix antennas using copper strips and 3D printing technology," IET Electronics Letters, vol. 52, no. 12, pp. 994-996, 2016.

[12] J. J. Adams, E. B. Duoss, T. F. Malkowski, M. J. Motala, B. Y. Ahn, R. G. Nuzzo, J. T. Bernhard, and J. A. Lewis, "Conformal printing of electrically small antennas on three-dimensional surfaces," Advanced Materials, vol. 23, no. 11, pp. 1335-1340, 2011.

[13] M. Kong, G. Shin, S. Lee, and I. J. Yoon, "An electrically small, 3D printed folded spherical meander antenna," in Proceedings of 2017 IEEE International Symposium on AsiaPacific Electromagnetic Compatibility (APEMC), Seoul, Korea, 2017, pp. 102-104.

[14] H. L. Thal, "New radiation Q limit for spherical wire antennas," IEEE Transaction on Antennas and Propagation, vol. 54, no. 10, pp. 2757-2763, 2006.

[15] Electrolube, "Silver Conductive Paint (SCP) technical data 
sheet," 2013; https://www.electrolube.com/core/components/ products/tds/044/SCP.pdf.

\section{Myeongjun Kong}

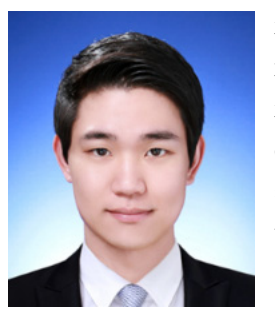

received his B.S. degree in Electrical Engineering from Chungnam National University, Daejeon, Korea in 2016. He is currently working toward his M.S. degree in Electrical Engineering at the same university. His research interests include antennas and electromagnetic compatibility.

\section{Geonyeong Shin}

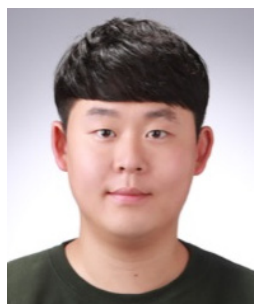

received his B.S. degree in Electrical Engineering from Chungnam National University, Daejeon, Korea in 2017. He is currently working toward his M.S. degree in Electrical Engineering at the same university. His research interests include antennas and RF/ microwave circuits.

[16] C. A. Balanis, Antenna Theory: Analysis and Design, 3rd ed. New York, NY: Wiley, 2005.

\section{Su-Hyeon Lee}

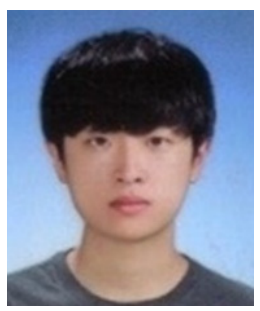

received his B.S. degree in Aerospace Engineering from Chonbuk National University, Jeonju, Korea in 2016. He is currently working toward his M.S. degree in Electrical Engineering at Chungnam National University, Daejeon, Korea. His research interests include antennas and electromagnetic wave propagation.

\section{Ick-Jae Yoon}

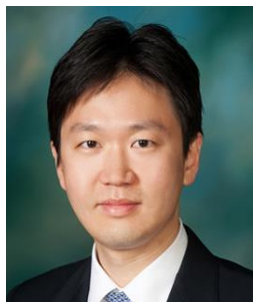

received his B.S. and M.S. degrees in Electrical Engineering from Yonsei University, Seoul, Korea in 2003 and 2005, respectively. He received his Ph.D. degree in Electrical Engineering from the University of Texas, Austin, TX, USA in 2012. He joined the faculty of Chungnam National University in Daejeon, Korea in September 2014 and is currently an Assistant Professor in the Department of Electrical Engineering. From 2012 to 2014, he was with the Electromagnetic Systems group of the Electrical Engineering Department at the Technical University of Denmark (DTU), Lyngby, DK as a Post-doctoral Research Fellow and an Assistant Professor. From 2005 to 2008, he worked as a Research Engineer at Samsung Advanced Institute of Technology of Samsung Electronics, Co., Yongin, Korea. His current research focuses on applied electromagnetics including antennas, $\mathrm{RF} /$ microwave circuits, electromagnetic compatibility, and theoretical methods for electromagnetics. Dr. Yoon received the H.C. Ørsted Post-doctoral Fellowship from DTU in 2012. 